\title{
LATTICES FOR MEDICAL SYNCHROTRONS EMPLOYING ACTIVE SCANNING
}

\author{
M. Benedikt, CERN/PS, CH-1211 Geneva 23, Switzerland
}

\begin{abstract}
Medical synchrotrons fall naturally into two categories; those using passive scattering techniques to spread the beam over the irradiation field and those using active scanning techniques to perform a high-precision conformal treatment of the tumour. The latter category is the newer generation that requires slower, more stable beam spills, achieved by the use of a third-integer extraction. These machines work below transition and for the stability of the stack, waiting outside the resonance, the chromaticity should be negative. This imposes strict constraints on the lattice for the application of the Hardt condition that aligns the separatrices of different momenta. This in turn affects the lattice between the electrostatic and magnetic septa, which should be, as close as possible, an achromatic transfer. It is also important that the extraction separatrices grow in a balanced way to economise the machine aperture. Since the separatrices occupy the same space at all energies, the 'good-field' region cannot be relaxed at higher energies as is normal in machines dominated by adiabatic damping. A sample lattice is presented to illustrate these points.
\end{abstract}

\section{INTRODUCTION}

Hadron-therapy can be based on protons or light ions. Extraction energies up to $400 \mathrm{MeV} / \mathrm{u}$ are needed for carbon ions, which requires a maximum magnetic rigidity of $6.5 \mathrm{Tm}$. Typically, such machines have a circumference of less than $80 \mathrm{~m}$ and small tune values. The use of a third-integer resonant extraction extends the beam spill time sufficiently to perform on-line dosimetry at the patient and to switch the beam on and off according to the dose required.

The uniformity of the spill, and the achieved dose distribution, depend critically on the method of extraction, on the lattice design and the stability of the power converters. It should also be remembered that such a machine will be in a hospital environment and therefore reliability, manoeuvrability of equipment and simplicity of operation will all be major concerns. The requirements on the spill are rather strict; the momentum spread of the extracted beam should be small, to make full use of the sharp dose localisation due to the Bragg peak, and the mean energy and the spread must not vary within a single spill, as this would cause a moving beam spot at the patient and an uncontrolled correlation between position and depth.

\section{BASIC LATTICE DESIGN}

The design shown below was developed from two achromatic, one-to-one mapping arcs $\left(\mu_{\mathrm{x}}=\mu_{\mathrm{z}}=2 \pi\right)$. Each arc bends $180^{\circ}$ and has a closed dispersion bump. This type of arc is extremely useful at the start of matching in a symmetric structure, as it will accept any Twiss input values and return them at the exit, always with the same phase advance. The arc was based on four $90^{\circ}$ FODO cells, in which the F-quadrupoles were later split to form a FOFODO cell with longer drift spaces after each pair of dipoles. Alternatively, this can be described as a series of triplets, in which the $\beta_{\mathrm{X}}$ is kept small and the vertical focusing of the rectangular dipoles is used to limit the peaks in $\beta_{\mathrm{z}}$. The smaller $\beta_{\mathrm{x}}$ is important at the maximum of the dispersion for the aperture and at the dipoles to limit the generation of the dispersion.

The arcs have then been detuned by changing the lengths of the drift sections to accommodate a betatron core, an internal dumping system and the electrostatic septum. The arcs are joined by two dispersion-free straight sections that are adjusted for the magnetic septum, the resonance sextupole and the rf and injection insertions. The lattice functions (Fig. 1) were adapted to the needs of each insertion with the help of a third quadrupole family, resulting in the final tune values of $Q_{\mathrm{x}}=1.67$ and $Q_{\mathrm{z}}=1.72$. The lattice has a superperiodicity of two with a mirror symmetry within each superperiod.

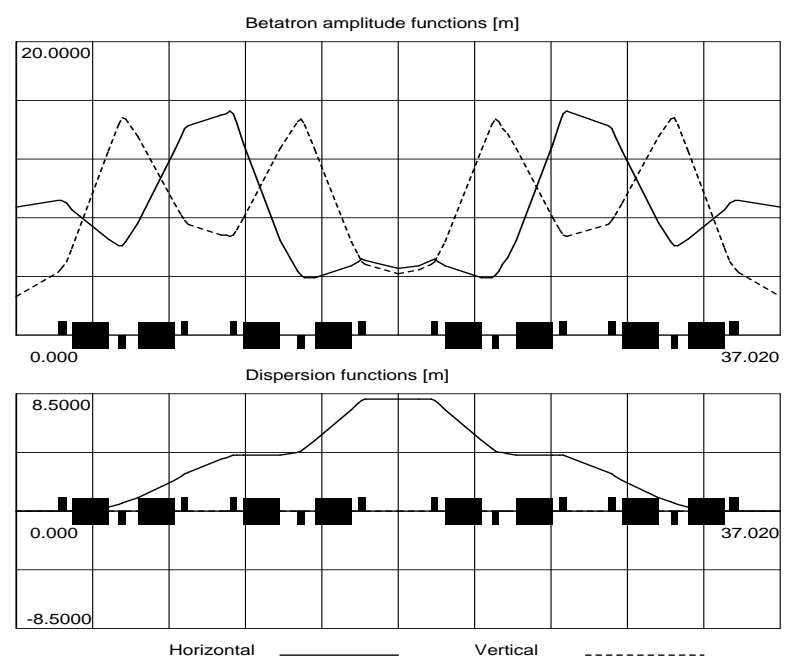

Figure 1: Lattice functions for one superperiod of the ring 
An advantage of the split F-structure is that the betafunctions are quasi constant in the long straight sections whereas in a FODO, they vary rapidly across drift spaces and tune changes can alter values at the extraction septa more easily. The smooth Twiss-functions avoid high focusing strengths. The quadrupoles are low-field, straight-pole units and the dipoles are H-type units with rectangular edge focusing, $1.85 \mathrm{~m}$ long. The sagitta of $\pm 4.5 \mathrm{~cm}$ is just acceptable in a straight magnet which has the advantage of being simple to construct.

\section{EXTRACTION}

\subsection{Sextupole magnets}

The main parameters that determine the phase-space geometry for a third-integer resonance are the chromaticity and the resonance excitation, both of which are controlled by sextupoles. To simplify operation, one would like to have independent handles on these two parameters. In the lattice shown, this is achieved by placing the resonance sextupole in one of the dispersionfree sections so that it has no chromatic effect. For an independent control of the chromaticity, the two-fold symmetry of the lattice and a horizontal tune close to an even number $\left(Q_{\mathrm{h}}=n \pm 1 / 3\right.$, with $n$ even) are used. Two sextupole magnets of equal strength are positioned in the dispersion region of the arcs on opposite sides of the ring, so that the resonance driving term is zero. The vertical chromaticity is adjusted in a similar way. To get some degree of orthogonality, the horizontal chromaticity sextupoles are at a large $\beta_{\mathrm{x}}$, small $\beta_{\mathrm{z}}$ and the converse applies to the vertical ones.

\subsection{Electrostatic Septum}

When the beam enters the resonance, there will be a range of momentum and emittance values that define a boundary between the stable and unstable regions, which is given by the width of the stop band for the third-integer resonance, as

$$
\left|Q_{x}^{\prime} \frac{\Delta p}{p}\right|=\sqrt{\varepsilon_{x}} \sqrt{\frac{1}{48 \sqrt{3} \pi}}|S|,
$$

where $S$ is the normalised resonance sextupole strength, $\varepsilon_{x}$ the horizontal emittance and $Q^{\prime}{ }_{\mathrm{x}}$ the absolute chromaticity, $\mathrm{d} Q_{\mathrm{x}} /(\mathrm{d} p / p)$. The dispersion function at the electrostatic septum will spread the separatrices so that particles with different momenta reach the septum with different angles. The condition to set the optics so that all the extraction separatrices are superimposed (Fig. 2) to give minimum losses on the electrostatic septum is known as the Hardt condition [1],

$$
D_{\mathrm{n}} \cos \alpha+D_{\mathrm{n}}^{\prime} \sin \alpha=-\frac{4 \pi}{S} Q_{x}^{\prime}
$$

where $D_{\mathrm{n}}$ and $D_{\mathrm{n}}^{\prime}$ are the normalised dispersion and its derivative and $\alpha$ is the angle measured from the $X$-axis in the anticlockwise direction to the perpendicular from the origin to the separatrix. For this condition, the normalised dispersion vector should be perpendicular to the separatrices. This then allows the superposition, using a large (absolute) chromaticity, which gives a small momentum spread for the extracted beam, as can be seen from (1). The large chromaticity also increases the tune spread of the beam, giving a defense against power supply ripple during extraction.

For a typical angle of $20^{\circ}$ to $50^{\circ}$ between the $X$-axis and the extraction separatices, $D_{\mathrm{n}}>0$ and $D_{\mathrm{n}}^{\prime}<0$ are needed (Fig. 2). The fact that negative chromaticity is required to ensure the stability of the stack [2], together with the above geometrical considerations, fixes the position of the electrostatic septum in the outer half of the vacuum chamber. This result is independent of whether the stack is positioned above or below the resonance in tune. In the lattice, the electrostatic septum is positioned $230^{\circ}$ downstream from the resonance sextupole in the long straight section in the second half of the arc, where $D_{\mathrm{n}}=1 \mathrm{~m}^{1 / 2}$ and $D_{\mathrm{n}}^{\prime}=-2 \mathrm{~m}^{1 / 2}$, as required for the Hardt condition. The angle between the extraction separatrices and the normalised dispersion vector is $80^{\circ}$ and the separatrices are superimposed for $Q^{\prime}{ }_{\mathrm{x}}=-4$.
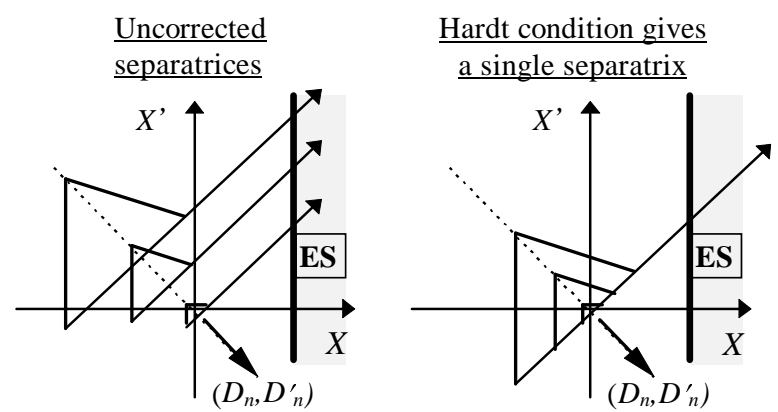

Figure 2: Extraction separatrices at electrostatic septum

\subsection{Magnetic Septum}

The magnetic extraction septum is positioned in the gap created by the kick of the electrostatic septum. In order to minimise the required voltage, both septa should be located at a large $\beta_{\mathrm{x}}$ and the phase separation should be close to $90^{\circ}$. A phase advance of $270^{\circ}$ is less convenient as it puts the magnetic septum to the inside and the extracted beam has to be transported through a longer distance, which in a small machine means that the crossing of a chromaticity sextupole is unavoidable.

The gap for the magnetic septum will be reduced due to the momentum spread of the extracted beam if the transfer from the electrostatic to the magnetic septum is not achromatic which is the case in the example lattice. To keep this effect small, the magnetic septum is best positioned in the dispersion free section just after the last dipole magnet of the arc. The phase advance between the 
septa is $55^{\circ}$, which gives $82 \%$ of the maximum gap opened by the kick of the electrostatic septum. The horizontal beta-functions at the septa are $\beta_{\mathrm{xES}}=15 \mathrm{~m}$ and $\beta_{\mathrm{xMS}}=9 \mathrm{~m}$, which with a kick of $2.5 \mathrm{mrad}$ gives a gap of $24 \mathrm{~mm}$. For an electrostatic septum of $1 \mathrm{~m}$ length, the corresponding voltage at top energy $\left(400 \mathrm{MeV} / \mathrm{u} \mathrm{C}^{6+}\right)$ is $34 \mathrm{kV} / \mathrm{cm}$. The gap reduction due to the non-achromatic transfer is $2.8(\Delta \mathrm{p} / \mathrm{p})[\mathrm{m}]$. For the example lattice, with a momentum spread of 0.001 , the reduced gap of $21 \mathrm{~mm}$ is still adequately wide to allow the magnetic septum to be placed outside the vacuum which improves the reliability of the system. The layout of the extraction elements is shown in Fig. 3.

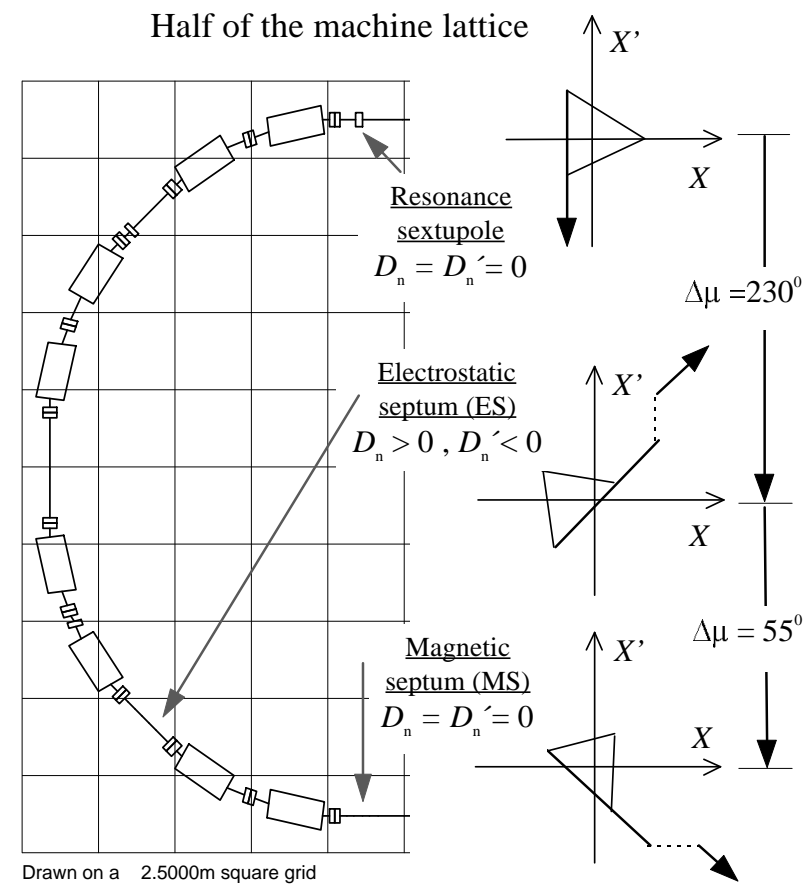

Figure 3: Layout of extraction elements

\subsection{Extraction method}

The choice of the extraction method will influence the spill characteristics. The Hardt condition is only effective if the resonance is stationary, i.e. if the beam is moved into the resonance e.g. by a betatron core $[3,4]$ or with rfnoise [5]. In this case, the beam is always extracted from the same radial position in the machine, with mean momentum and momentum spread remaining unchanged during the spill. Therefore no dynamic orbit, focusing or tune corrections are required. The time structure of the spill can be improved by increasing the velocity in tune with which particles enter the resonance. One possibility is to slowly accelerate an unbunched beam with a large momentum spread into the resonance and to front-end this slow acceleration with an empty rf-bucket positioned over the resonance [6].

\section{CONCLUSIONS}

In any machine design there are essential requirements and desirable features. Inevitably there are conflicts, and compromises are needed. For example, reducing the dipole gap height often has high priority on grounds of power, whereas in a medical synchrotron the power dissipation is less important than optimum lattice functions and low-ripple power converters for the extraction. The lattice presented meets the extraction requirements. The only compromise is the transfer from the electrostatic to magnetic septum, which is not entirely achromatic but the required kick is very modest and there is no problem in opening sufficient gap for the magnetic septum. The resonance is stationary during extraction, as are all transverse beam parameters. This is an essential requirement for a stable spill since it avoids the need for dynamic corrections of orbits, tuning and resonance excitation, and delivers a beam with constant energy and momentum spread.

\section{ACKNOWLEDGMENTS}

This work constitutes a preparatory lattice design for a medical synchrotron and was carried out within the framework of the Proton Ion Medical Machine Study (PIMMS), which is a collaboration between CERN, GSI, Med-AUSTRON and TERA that is hosted by the PSDivision in CERN. The author would like to thank the members of the collaboration for many helpful discussions and Phil Bryant for introducing him to lattice design.

\section{REFERENCES}

[1] W. Hardt, Ultraslow extraction out of Lear (transverse aspects), CERN, internal report PS/DL/LEAR Note 81-6.

[2] W. Schnell, B. Zotter, A simplified criterion for transverse stability of a coasting beam, and its application to the ISR, CERN-ISR-GS-RF/76-26

[3] L. Badano, S. Rossi, Characteristics of a betatron core for extraction in a proton-ion medical synchrotron, to be published.

[4] J.C Ciret, Extraction du faisceau de Saturne II par acceleration betatronique. Le Gephyrotron, GERMA 76.02/IE-117.

[5] S. van der Meer, Stochastic extraction, A low ripple version of resonant extraction, CERN/PS/AA, 78-6

[6] R. Cappi, Ch. Steinbach, Improvement of the low frequency duty factor of slow extraction by $\mathrm{rf}$ phase displacement, PAC 1981, Washington. 